\title{
Derivations of a Sullivan Model and the Rationalized G-Sequence
}

\author{
Oteng Maphane \\ Department of Mathematics and Statistical Sciences, Faculty of Science, \\ Botswana International University of Science and Technology, Palapye, Botswana \\ Correspondence should be addressed to Oteng Maphane; maphaneot@biust.ac.bw
}

Received 2 November 2020; Accepted 12 January 2021; Published 1 February 2021

Academic Editor: Sumit Chandok

Copyright (C) 2021 Oteng Maphane. This is an open access article distributed under the Creative Commons Attribution License, which permits unrestricted use, distribution, and reproduction in any medium, provided the original work is properly cited.

Let $G_{k, n}(\mathbb{C})$ for $2 \leq k<n$ denote the Grassmann manifold of $k$-dimensional vector subspaces of $\mathbb{C}^{n}$. In this paper, we compute, in terms of the Sullivan models, the rational evaluation subgroups and, more generally, the $G$-sequence of the inclusion $G_{2, n}(\mathbb{C}) \mapsto G_{2, n+r}(\mathbb{C})$ for $r \geq 1$.

\section{Introduction}

Throughout this paper, we rely on the theory of minimal Sullivan models in rational homotopy theory for which [1] is our standard reference. Let $X$ be a based CW-complex; the $n$th Gottlieb group of $X$ (or the $n$th evaluation subgroup [2] of $\pi_{n}(X)$ ), denoted by $G_{n}(X)$, consists of those elements $a \in \pi_{n}(X)$ for which there is a continuous map $H: X \times$ $S^{n} \longrightarrow X$ such that the following diagram commutes:

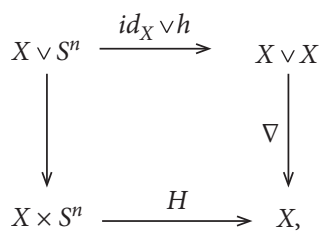

where $h: S^{n} \longrightarrow X$ is a representative of $a$ and $\nabla$ is the folding map. Let $f: X \longrightarrow Y$ be a based map of simply connected finite $\mathrm{CW}$-complexes. In [3], the evaluation at the base point of $X$ gives the evaluation map $\omega$ : (Map) $(\mathrm{X}, \mathrm{Y} ; \mathrm{f}) \longrightarrow \mathrm{Y}$, where (Map) $(\mathrm{X}, \mathrm{Y} ; \mathrm{f})$ is the component of $f$ in the space of mappings from $X$ to $Y$. The image of the homomorphism induced in homotopy groups

$$
\omega_{\sharp}: \pi_{*}(\operatorname{Map}(X, Y ; f)) \longrightarrow \pi_{*}(Y)
$$

is called the $n$th evaluation subgroup of $p$, and it is denoted by $G_{n}(Y, X ; f)$. Moreover, if $f=i d_{X}$, the space (Map) $(\mathrm{X}, \mathrm{Y} ; \mathrm{f})$ is the monoid aut ${ }_{1}(X)$ of self-equivalences of $X$ homotopic to the identity of $X$; then, $e v$ : $\operatorname{aut}_{1}(X) \longrightarrow X$ is the evaluation map, and the image of the induced homomorphism

$$
e v_{\sharp}: \pi_{*}\left(\operatorname{aut}_{1}(X)\right) \longrightarrow \pi_{*}(X)
$$

is $G_{n}(X)$, i.e., the $n$th Gottlieb group. Moreover, in [4], Woo and Lee studied the relative evaluation subgroups $G_{n}^{\text {rel }}(X, Y ; p)$ and proved that they fit in a sequence

$$
\cdots \longrightarrow G_{n+1}^{\mathrm{rel}}(X, Y ; f) \longrightarrow G_{n}(X) \longrightarrow G_{n}(X, Y ; f) \longrightarrow \cdots
$$

called the $G$-sequence of $f$. Finally, in [3], Smith and Lupton identify the homomorphism induced on rational homotopy groups by the evaluation map $\omega:(\operatorname{Map}(X, Y ; f)) \longrightarrow \mathrm{Y}$, in terms of a map of complexes of derivations constructed directly from the Sullivan minimal model of $f$. In [5], the authors use a map of complexes of derivations of minimal Sullivan models of mapping spaces to compute rational relative Gottlieb groups of the inclusion $G_{2, n}(\mathbb{C}) \rightarrow G_{2, n+1}(\mathbb{C})$ between complex Grassmannians. In this paper, we generalize their work to compute rational relative Gottlieb groups of the inclusion $G_{k, n}(\mathbb{C}) \mapsto G_{k, n+r}$ (C), $r \geq 1$ between complex Grassmannians for $2 \leq k<n$.

\section{Preliminaries}

Here, we fix terminology and recall some standard facts on differential graded algebras. All vector spaces and algebras are taken over a field $\mathbb{Q}$ of rational numbers. 
Definition 1. A graded algebra $A$ is a sum $A=\oplus_{n \geq 0}$ $A^{n}$, where $A^{n}$ is a vector space, together with an associative multiplication $A^{i} \otimes A^{j} \longrightarrow A^{i+j}, x \otimes y \mapsto x y$, and has $1 \in A^{0}$. It is graded commutative if, for any homogeneous elements $x$ and $y$,

$$
x y=(-1)^{|x||y|} y x,
$$

where $|x|=i$ for $x \in A^{i}$. If $A$ is a graded algebra equipped with a linear differential map $d: A^{n} \longrightarrow A^{n+1}$ such that $d^{\circ} d=0$ and

$$
d(x y)=(d x) y+(-1)^{|x|} x(d y)
$$

then $(A, d)$ is called a differential-graded algebra and $d$ is called a differential. Moreover, if $A$ is also a graded commutative algebra, then $(A, d)$ is a commutative differential graded algebra (cdga). It is said to be connected if $A^{0} \cong \mathbb{Q}$.

Definition 2. Let $V=\oplus_{i \geq 0} V^{i}$ with $V^{\text {even }}:=\oplus_{i \geq 0} V^{2 i}$ and $V^{\text {odd }}:=\oplus_{i \geq 1} V^{2 i-1}$. A commutative-graded algebra $A$ is called free commutative if $A=\wedge V=S\left(V^{\text {even }}\right) \otimes E\left(V^{\text {odd }}\right)$, where $S\left(V^{\text {even }}\right)$ is the symmetric algebra on $V^{\text {even }}$ and $E\left(V^{\text {odd }}\right)$ is the exterior algebra on $V^{\text {odd }}$.

Definition 3. A Sullivan algebra is a commutative differential-graded algebra $(\wedge V, d)$, where $V=\cup_{k>0} V(k)$ and $V(0) \subset V(1) \cdots$ such that $d V(0)=0$ and $d V(k) \subset \wedge V(k-$ 1). It is called minimal if $d V \subset \wedge^{\geq 2} V$.

If $(A, d)$ is a cdga whose cohomology is connected and finite dimensional in each degree, then there always exists a quasi-isomorphism from a Sullivan algebra $(\wedge V, d)$ to $(A, d)$ [1]. To each simply connected space, Sullivan associates a cdga $A_{\mathrm{PL}}(X)$ of rational polynomial differential forms on $X$ that uniquely determines the rational homotopy type of $X$ [6]. A minimal Sullivan model of $X$ is a minimal Sullivan model of $A_{\mathrm{PL}}(X)$. More precisely, $H^{*}(\wedge V, d) \cong H^{*}(X ; \mathbb{Q})$ as graded algebras and $V \cong \pi_{*}(X) \otimes \mathbb{Q}$ as graded vector spaces.

\section{Derivations of a Sullivan Model and the G- Sequence}

Let $(A, d)$ be a commutative differential-graded algebra. A derivation $\theta$ of degree $k$ is a linear mapping $\theta: A^{n} \longrightarrow A^{n-k}$ such that $\theta(a b)=\theta(a) b+(-1)^{k|a|} a \theta(b)$. Denote by $\operatorname{Der}_{k} A$ the vector space of all derivation of degree $k$ and $\operatorname{Der} A=$ $\oplus_{k} \operatorname{Der}_{k} A$. The commutator bracket induces a graded Lie algebra structure on $\operatorname{Der} A$. Moreover, $(\operatorname{Der} A, \delta)$ is a differential graded Lie algebra [6], with the differential $\delta$ defined in the usual way by

$$
\delta \theta=d \circ \theta+(-1)^{k+1} \theta \circ d .
$$

Let $(\wedge V, d)$ be a Sullivan algebra, where $V$ is spanned by $\left\{v_{1}, \ldots, v_{k}\right\}$. Then, $\operatorname{Der} \wedge V$ is spanned by $\theta_{1}, \ldots, \theta_{k}$, where $\theta_{i}$ is the unique derivation of $\wedge V$ defined by $\theta_{i}\left(v_{j}\right)=\delta_{i j}$. The derivation $\theta_{i}$ will be denoted by $\left(v_{i}, 1\right)$. Moreover, an element $v \in V \cong \pi_{*}(X) \otimes \mathbb{Q}$ is a Gottlieb element of $\pi_{*}(X) \otimes \mathbb{Q}$ if and only if there is a derivation $\theta$ of $\wedge V$ satisfying $\theta(v)=1$ and such that $\delta \theta=0$, see page 392 in [1].

Let $\phi:(A, d) \longrightarrow(B, d)$ be a morphism of cdga's. A $\phi$-derivation of degree $k$ is a linear mapping $\theta: A^{n} \longrightarrow B^{n-k}$ for which $\theta(a b)=\theta(a) \phi(b)+(-1)^{k|a|} \phi(a) \theta(b)$.

We consider only derivations of positive degree. Denote by $\operatorname{Der}_{n}(A, B ; \phi)$ the vector space of $\phi$-derivations of degree $n$ for $n>0$ and by $\operatorname{Der}(A, B ; \phi)=\oplus_{n} \operatorname{Der}_{n}(A, B ; \phi)$ the $\mathbb{Z}$-graded vector space of all $\phi$-derivations. The differentialgraded vector space of $\phi$-derivations is denoted by $(\operatorname{Der}(A, B ; \phi), \partial)$, where the differential $\partial$ is defined by $\partial \theta=$ $d_{B}{ }^{\circ} \theta+(-1)^{\mathrm{k}+1} \theta^{\circ} \mathrm{d}_{\mathrm{A}}$. In case $A=B$ and $\phi=1_{B}$, then $(\operatorname{Der}(B, B ; 1), \partial)$ is just the usual differential-graded Lie algebra of derivations on the cdga $B$ [3]. Whenever $A=$ $(\wedge V, d)$ is a Sullivan algebra, we note that there is an isomorphism of graded vector spaces:

$$
\operatorname{Der}(A, B ; \phi) \cong \operatorname{Hom}(V, B)
$$

If $\left\{v_{i}\right\}$ is a basis of $V$, then the vector space $\operatorname{Der}(A, B ; \phi)$ is spanned by the unique $\phi$-derivation $\theta$ denoted by $\left(v_{i}, b_{i}\right)$ such that $\theta_{i}\left(v_{i}\right)=b_{i}$, where $b_{i} \in B$ and $\theta_{i}\left(v_{j}\right)=0$ for $i \neq j$. Moreover, in [3], precomposition with $\phi$ gives a chain complex map $\phi^{*}: \operatorname{Der}(B, B ; 1) \longrightarrow \operatorname{Der}(A, B ; \phi)$ and postcomposition with the augmentation $\varepsilon: B \longrightarrow \mathbb{Q}$ gives a chain complex map $\varepsilon_{*}: \operatorname{Der}(A, B ; \phi) \longrightarrow \operatorname{Der}(A, \mathbb{Q} ; \varepsilon)$. The evaluation subgroup of $\phi$ is defined as follows:

$$
\begin{aligned}
G_{n} & (A, B ; \phi) \\
\quad & =\operatorname{Im}\left\{H\left(\varepsilon_{*}\right): H_{n}(\operatorname{Der}(A, B ; \phi)) \longrightarrow H_{n}(\operatorname{Der}(A, \mathbb{Q} ; \varepsilon))\right\} .
\end{aligned}
$$

In case $A=B$ and $\phi=1_{B}$, we get the Gottlieb group of $(B, d)$ defined as follows:

$$
\begin{aligned}
G_{n}(B) & =\operatorname{Im}\left\{H\left(\varepsilon_{*}\right): H_{n}(\operatorname{Der}(B, B ; 1))\right. \\
& \left.\longrightarrow H_{n}(\operatorname{Der}(B, \mathbb{Q} ; \varepsilon))\right\} .
\end{aligned}
$$

In particular, $G_{n}(B) \cong G_{n}\left(X_{\mathbb{Q}}\right)$, if $B$ is the minimal Sullivan model of a simply connected space $X$ (see Proposition 29.8 in [1]).

Definition 4 (see $[3,7])$. Let $\phi: A \longrightarrow B$ be a map of differential-graded vector spaces. A differential-graded vector space, $\operatorname{Rel}_{*}(\phi)$, called the mapping cone of $\phi$ is defined as follows. $\operatorname{Rel}_{n}(\phi)=A_{n-1} \oplus B_{n}$ with the differential $\delta(a, b)=\left(-d_{A}(a), \phi(a)+d_{B}(b)\right)$. There are inclusion and projection chain maps $J: B_{n} \longrightarrow \operatorname{Rel}_{n}(\phi)$ and $P: \operatorname{Rel}_{n}(\phi) \longrightarrow A_{n-1}$ defined by $J(w)=(0, w)$ and $P(a, b)=a$. These yields a short exact sequence of chain complexes

$$
0 \longrightarrow B_{*} \stackrel{J}{\longrightarrow} \operatorname{Rel}_{*}(\phi) \stackrel{P}{\longrightarrow} A_{*-1} \longrightarrow 0,
$$

and a long exact homology sequence of $\phi$ 


$$
\cdots \longrightarrow H_{n+1}(\operatorname{Rel}(\phi)) \stackrel{H}{\longrightarrow}(P) H_{n}(A) \stackrel{H}{\longrightarrow}(\phi) H_{n}(B) \stackrel{H}{\longrightarrow}(J) H_{n}(\operatorname{Rel}(\phi)) \longrightarrow \cdots
$$

whose connecting homomorphism is $H(\phi)$.

Following [3], we consider a commutative diagram of differential-graded vector spaces:

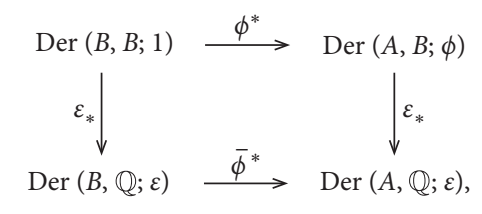

where $\varepsilon$ is the augmentation of either $A$ or $B$, which leads to the following homology ladder for $n \geq 2$ :

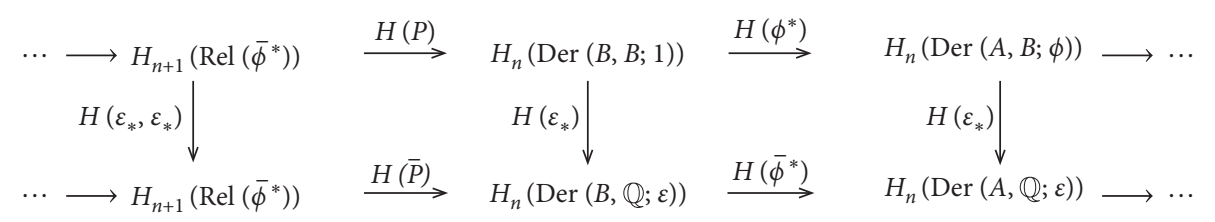
follows:

The $n$th relative evaluation subgroup of $\phi$ is defined as

The $G$-sequence of the map $\phi: A \longrightarrow B$ is given by

$$
G_{n}^{r e l}=\operatorname{Im}\left\{H\left(\varepsilon_{*}, \varepsilon_{*}\right): H_{n}\left(\operatorname{Rel}\left(\phi^{*}\right)\right) \longrightarrow H_{n}\left(\operatorname{Rel}\left(\bar{\phi}^{*}\right)\right)\right\} .
$$

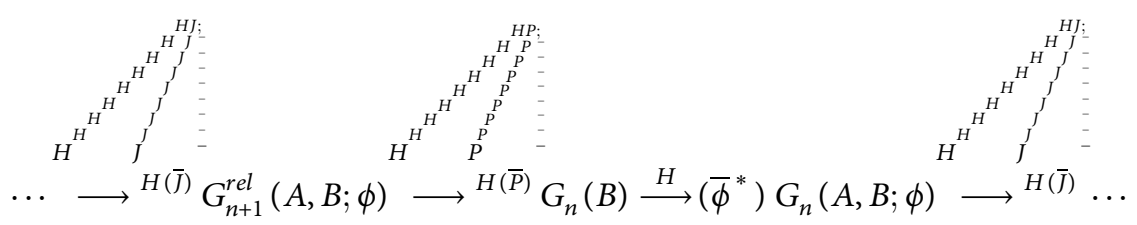

which ends in $G_{2}(A, B ; \phi)$. Moreover, Theorem 3.5 in [3] can be applied to the Sullivan model $\phi:(\wedge V, d) \longrightarrow(B, d)$ of the map $f: X \longrightarrow Y$.

\section{The Inclusion $G_{k, n}(\mathbb{C}) \longmapsto G_{k, n+1}(\mathbb{C})$}

The complex Grassmannian $G_{k, n}(\mathbb{C})$ is a simply connected homogeneous space as $G_{k, n}(\mathbb{C}) \cong U(n) /(U(k) \times U(n-k))$ for $1 \leq k<n$, where $U(n)$ is the unitary group. It is a symplectic manifold of dimension $2 m$, where $m=k(n-k)$. As the complex Grassmannian $G_{k, n}(\mathbb{C})$ is simply connected, so we may associate a minimal Sullivan model.

The method to compute a Sullivan model of the homogeneous space $G_{k, n}(\mathbb{C})$ is given in detail in $[8,9]$.

Following [9], a Sullivan model of $G_{k, n}(\mathbb{C})$ for $1 \leq k<n$ is given by

$$
\left(\wedge\left(b_{2}, b_{4}, \ldots, b_{2 k}, x_{2}, x_{4}, \ldots, x_{2(n-k)}, y_{1}, y_{3}, \ldots, y_{2 n-1}\right), d\right),
$$

with

$$
d b_{i}=0=d x_{j}, d y_{2 p-1}=\sum_{p_{1}+p_{2}=p} b_{2 p_{1}} x_{2 p_{2}}, 1 \leq p \leq n .
$$

Lemma 1. The minimal Sullivan model of $G_{k, n}(\mathbb{C})$ for $2 \leq k<n$ is given by

$$
\left(\wedge\left(b_{2}, \ldots, b_{2 k}, y_{2(n-k)+1}, \ldots, y_{2 n-1}\right), d\right),
$$

where $d b_{i}=0$ and $d y_{2(n-k)+1} \in \wedge\left(b_{2}, \ldots, b_{2 k}\right)$.

Proof. Consider the Sullivan model

$$
\left(\wedge\left(b_{2}, b_{4}, \ldots, b_{2 k}, x_{2}, x_{4}, \ldots, x_{2(n-k)}, y_{1}, y_{3}, \ldots, y_{2 n-1}\right), d\right),
$$

of $G_{k, n}(\mathbb{C})$ for $2 \leq k<n$ : 


$$
\begin{aligned}
d y_{1}= & b_{2}+x_{2}, \\
d y_{3}= & b_{4}+x_{4}+b_{2} x_{2}, \\
& \vdots \\
d y_{2 n-1}= & b_{2 k} x_{2(n-k)} .
\end{aligned}
$$

The model is not minimal as the linear part is not zero. To find its minimal Sullivan model, we make a change of variable $t_{2}=b_{2}+x_{2}$ and replace $x_{2}$ by $t_{2}-b_{2}$ wherever it appears in the differential. This gives an isomorphic Sullivan algebra

$$
\left(\wedge\left(b_{2}, t_{2}, b_{4}, \ldots, b_{2 k}, x_{4}, \ldots, x_{2(n-k)}, y_{1}, y_{3}, \ldots, y_{2 n-1}\right), d\right),
$$

where

$$
\begin{gathered}
d y_{1}=t_{2}, \\
d y_{3}=b_{4}+x_{4}+b_{2}\left(t_{2}-b_{2}\right), \\
\vdots \\
d y_{2 n-1}=b_{2 k} x_{2(n-k)} .
\end{gathered}
$$

As the ideal generated by $y_{1}$ and $t_{2}$ is acyclic, the above Sullivan algebra is quasi-isomorphic to

$$
\left(\wedge\left(b_{2}, b_{4}, \ldots, b_{2 k}, x_{4}, \ldots, x_{2(n-k)}, y_{3}, \ldots, y_{2 n-1}\right), d\right)
$$

where

$$
\begin{gathered}
d y_{3}=b_{4}+x_{4}-b_{2}^{2}, \\
\vdots \\
d y_{2 n-1}=b_{2 k} x_{2(n-k)} .
\end{gathered}
$$

One continues in this fashion and makes another change of variable $t_{4}=b_{4}+x_{4}-b_{2}^{2}$ and replaces $x_{4}$ by $t_{4}-b_{4}+b_{2}^{2}$ wherever it appears in the differential and does so until they reach a change of variable of the form

$$
\begin{array}{r}
t_{2(n-k)}=b_{2(n-k)}+x_{2(n-k)}+\alpha \text { for } n=2 k \\
\quad \text { or } t_{2(n-k)}=x_{2(n-k)}+\beta \text { for } n>2 k,
\end{array}
$$

where $\alpha \in \wedge\left(b_{2}, \ldots, b_{2(k-1)}\right), \beta \in \wedge\left(b_{2}, \ldots, b_{2 k}\right)$ and replace

$$
x_{2(n-k)}= \begin{cases}t_{2(n-k)}-b_{2 k}+\alpha, & \text { for } n=2 k, \\ t_{2(n-k)}+\beta, & \text { for } n>2 k,\end{cases}
$$

wherever it appears in the differential. This gives an isomorphic Sullivan algebra:

$$
\left(\wedge\left(b_{2}, \ldots, b_{2 k}, y_{2(n-k)-1}, y_{2(n-k)+1}, \ldots, y_{2 n-1}\right), d\right),
$$

where

$$
\begin{aligned}
& d y_{2(n-k)-1}= t_{2(n-k)}, \\
& \vdots \\
& d y_{2 n-1}=b_{2 k} x_{2(n-k)} .
\end{aligned}
$$

As the ideal generated by $t_{2(n-k)}$ and $y_{2(n-k)-1}$ is acyclic, we get the minimal Sullivan model:

$$
\left(\wedge\left(b_{2}, \ldots, b_{2 k}, y_{2(n-k)+1}, \ldots, y_{2 n-1}\right), d\right),
$$

with $d b_{i}=0$ and $d y_{2(n-k)+1} \in \wedge\left(b_{2}, \ldots, b_{2 k}\right)$.

In the same way, by Lemma 1 , the minimal Sullivan model of $G_{k, n+r}(\mathbb{C})$ for $2 \leq k<n+r$ and $r \geq 1$ is given by

$$
\left(\wedge\left(a_{2}, \ldots, a_{2 k}, z_{2(n+r-k)+1}, \ldots, z_{2(n+r)-1}\right), d\right),
$$

where $d a_{i}=0$ and $d z_{2(n+r-k)+1} \in \wedge\left(a_{2}, \ldots, a_{2 k}\right)$. We establish the following results.

Theorem 1. Let $B=\left(\wedge\left(b_{2}, \ldots, b_{2 k}, y_{2(n-k)+1}, \ldots, y_{2 n-1}\right), d\right)$. Then, $G_{n}(B)=\left\langle\left[y_{2(n-k)+1}^{*}\right], \ldots,\left[y_{2 n-1}^{*}\right]\right\rangle$.

Proof. Let $\left(y_{2(n-t)+1}, 1\right)$ denote the derivation $\theta_{2(n-t)+1}$ for $t \in\{1, \ldots, k\}$ such that $\theta_{2(n-t)+1}\left(y_{2(n-t)+1}\right)=1$ and zero on other generators. Then,

$$
\delta \theta_{2(n-t)+1}\left(y_{2(n-t)+1}\right)=0 \text {. }
$$

Moreover, the generators $\theta_{2(n-t)+1}$ cannot be boundaries for degree reasons. Therefore, $\left[\theta_{2(n-t)+1}\right]$ are nonzero homology classes in $H_{*}(\operatorname{Der}(B, B ; 1))$. Furthermore, $\mathcal{E}_{*}\left(\theta_{2(n-t)+1}\right)=y_{2(n-t)+1}^{*}$.

As $G_{k, n}(\mathbb{C})$ is a simply connected finite CW-complex, then $G_{\text {even }}(B)=0$ (see Proposition 28.8 in [1]. Thus, $G_{n}(B)=\left\langle\left[y_{2(n-k)+1}^{*}\right], \ldots,\left[y_{2 n-1}^{*}\right]\right\rangle$.

Theorem 2. Given the inclusion $G_{k, n}(\mathbb{C}) \mapsto G_{k, n+r}(\mathbb{C}), r \geq 1$ for $2 \leq k<n$ and $\phi:(\wedge V, d) \longrightarrow(B, d)$ for its Sullivan model, then $G_{*}(\wedge V, B ; \phi)=\left\langle\left[z_{2(n+r-k)+1}^{*}\right], \ldots,\left[z_{2(n+r)-1}^{*}\right]\right\rangle$.

Proof. The vector space $\operatorname{Der}(\wedge V, B ; \phi)$ is generated by the derivations $\theta_{2(n+r-t)+1}=\left(z_{2(n+r-t)+1}, 1\right)$ for $t \in\{1, \ldots, k\}$. The differential is given by

$$
\delta \theta_{2(n+r-t)+1}\left(z_{2(n+r-t)+1}\right)=0 .
$$

Hence, $\left[\theta_{2(n+r-t)+1}\right]$ are the nonzero homology classes in $H_{*}(\operatorname{Der}(\wedge V, B ; \phi))$. Moreover, $H\left(\varepsilon_{*}\right)\left(\left[\theta_{2(n+r-t)+1}\right]\right)=$ $\left[z_{2(n+r-t)+1}^{*}\right]$, where $z_{2(n+r-t)+1}^{*} \in \operatorname{Der}(\wedge V, \mathbb{Q}, \varepsilon)$. Thus, $G_{*}(\wedge V, B ; \phi)=\left\langle\left[z_{2(n+r-k)+1}^{*}\right] \ldots,\left[z_{2(n+r)-1}^{*}\right]\right\rangle$.

Theorem 3. Given the inclusion $G_{k, n}(\mathbb{C}) \mapsto G_{k, n+r}(\mathbb{C}), r \geq 1$ for $2 \leq k<n$ and $\phi:(\wedge V, d) \longrightarrow(B, d)$ for its Sullivan model, then $G_{*}^{r e l}(\wedge V, B ; \phi)=\left\langle\left[\left(y_{2(n-k)+1}^{*}, 0\right)\right],\left[\left(0, z_{2(n+r)-1}^{*}\right)\right]\right\rangle$.

Proof. Define the derivations $\alpha_{2(n-t)+1}=\left(y_{2(n-t)+1}, 1\right)$ for $t \in\{1, \ldots, k\}$ in $\operatorname{Der}(B, B ; 1)$ and $\theta_{2(n+r-t)+1}=\left(z_{2(n+r-t)+1}, 1\right)$ in $\operatorname{Der}(\wedge V, B ; \phi)$. Then,

$$
\phi^{*}\left(\alpha_{2(n-t)+1}\right)= \begin{cases}\theta_{2(n+r-t)+1}, & \text { if } 2(n-t)+1=2(n+r-t)+1, \\ 0, & \text { if } 2(n-t)+1 \neq 2(n+r-t)+1,\end{cases}
$$

such that 


$$
D\left(\alpha_{2(n-t)+1}, 0\right)= \begin{cases}\left(0, \theta_{2(n+r-t)+1}\right), & \text { if } 2(n-t)+1=2(n+r-t)+1, \\ (0,0), & \text { if } 2(n-t)+1 \neq 2(n+r-t)+1\end{cases}
$$

and $D\left(0, \theta_{2(n+r-t)+1}\right)=(0,0)$. Thus, $\left[\left(\alpha_{2(n-t)+1}, 0\right)\right]$ if $2(n-$ $t)+1 \neq 2(n+r-t)+1$ and $\left[\left(0, \theta_{2(n+r-t)+1}\right)\right]$ are the nonzero homology classes in $H_{*}\left(\operatorname{Rel}\left(\phi^{*}\right)\right)$. Moreover, $H\left(\varepsilon_{*}, \varepsilon_{*}\right)\left(\left[\left(\alpha_{2(n-t)+1}, 0\right)\right]\right)=\left[\left(y_{2(n-t)+1}^{*}, 0\right)\right]$, for $2(n-t)+$ $1 \neq 2(n+r-t)+1$, and in the same way,
$H\left(\varepsilon_{*}, \varepsilon_{*}\right)\left(\left[\left(0, \theta_{2(n+r-t)+1}\right)\right]\right)=\left[\left(0, z_{2(n+r-t)+1}^{*}\right)\right]$. A straightforward calculation shows that $\left[\left(y_{2(n-t)+1}^{*}, 0\right)\right]$ for $2(n-t)+$ $1 \neq 2(n+r-t)+1$ and $\left[\left(0, z_{2(n+r-t)+1}^{*}\right)\right] \operatorname{span} H\left(\varepsilon_{*}, \varepsilon_{*}\right)$.

The $G$-sequence reduces to

$$
\begin{aligned}
& 0 \longrightarrow G_{2(n+r)-1}(\wedge V, B ; \phi) \stackrel{H(J)}{\simeq} G_{2(n+r)-1}^{\mathrm{rel}}(\wedge V, B ; \phi) \longrightarrow 0, \\
& 0 \longrightarrow G_{2(n-t)+1=2(n+r-t)+1}(B) \stackrel{H\left(\phi^{*}\right)}{\longrightarrow} G_{2(n-t)+1=2(n+r-t)+1}(\wedge V, B ; \phi) \longrightarrow 0, \\
& 0 \longrightarrow G_{2(n-k)+1}^{\mathrm{rel}}(\wedge V, B ; \phi) \stackrel{H(P)}{\simeq} G_{2(n-k)+1}(B) \longrightarrow 0,
\end{aligned}
$$

and it is exact.

\section{Data Availability}

No data were used to support this study.

\section{Conflicts of Interest}

The author declares no conflicts of interest.

\section{Acknowledgments}

This work was completed with the support of the Botswana International University of Science and Technology (BIUST).

\section{References}

[1] Y. Félix, S. Halperin, and J. C. Thomas, Rational Homotopy Theory, Springer, New York, NY, USA, 2001.

[2] D. H. Gottlieb, "Evaluation subgroups of homotopy groups," American Journal of Mathematics, vol. 91, no. 3, pp. 729-756, 1969.

[3] G. Lupton and S. B. Smith, "Rationalized evaluation subgroups of a map I: Sullivan models, derivations and G-sequences," Journal of Pure and Applied Algebra, vol. 209, no. 1, pp. 159-171, 2007.

[4] M. H. Woo and K. Y. Lee, "On the relative evaluation subgroups of a CW-pair," Journal of the Korean Mathematical Society, vol. 25, no. 1, pp. 149-160, 1988.

[5] P. A. Otieno, J.-B. Gatsinzi, and V. O. Otieno, "Rationalized evaluation subgroups of mapping spaces between complex Grassmannians," Afrika Matematika, vol. 31, pp. 297-303, 2020.

[6] D. Sullivan, "Infinitesimal computations in topology," Publications Mathématiques de l'I.H.É.S, vol. 47, pp. 269-331, 1977.

[7] E. H. Spanier, Algebraic Topology, Corrected reprint of the 1966 original, Springer-Verlag, New York, NY, USA, 1989.

[8] W. Greub, S. Halperin, and R. Vanstone, Connections, Curvature, and Cohomology, Vol. III, Academic Press, New York, NY, USA, 1976.

[9] A. Murillo, "The top cohomology class of classical compact homogeneous spaces," Algebras Groups and Geometries, vol. 16, pp. 531-550, 1999. 